\title{
REFLECTION-IMPULSIVITY IN PERCEPTUAL AND CONCEPTUAL MATCHING TASKS AMONG KINDERGARTEN GHILDREN
}

\author{
GIYOO HATANO AND KAYOKO INAGAKI ${ }^{1}$ \\ Dokkyo University Izumi Junior College
}

\begin{abstract}
In order to examine the generality of reflection-impulsivity, 51 5-6-year-olds were given, in addition to Kagan's MFF, a tactual-visual matching task (TVM) of geometric shapes and two conceptual matching tasks: Referential communication with inadequate message where the child as receiver had to ask questions to make a correct choice, and a revised version of Olson's conceptual pattern matching. Latency and error measures of MFF and TVM were all significantly correlated to each other. Among the 24 correlations between these 4 measures from the perceptual tasks and 3 measures each from conceptual ones, i.e., amount of instrumental information-seeking, number of errors and number of guesses, 21 had the expected signs $(+,-)$ and 9 were significant in the expected direction. Partialing out verbal ability made only 2 of the 9 insignificant.
\end{abstract}

The present study was aimed at investigating whether reflection-impulsivity exists as a general trait among kindergarten children. According to Kagan and Kogan (1970), this dimension of cognitive style is defined as the degree to which a subject reflects on the validity of his solution hypotheses. One of the implications of this conceptualization is that, when the subject is allowed to make as many instrumental information-seeking responses preceding a choice or decision response as he wants, the number of such instrumental responses and number of erroneous choice responses, are expressions of his reflectionimpulsivity.

Reflective and impulsive children are

${ }^{1}$ We are grateful to Mr. Keigo Takahashi, director of Dokanyama Kindergarten for his making the subjects available to us, and to Ms. Etsuko Hatano and Ms. Naomi Sakata for their assistance in conducting the experiment. We would also like to thank Mr. Christpher W. P. Reynolds for his help in preparing this English draft.

Request for reprints should be sent to $G$. Hatano, Dokkyo University, 600 Sakae-cho, Soka-shi, Saitama, 340 Japan. usually classified by considering both latency and error measures in the Matching Familiar Figures Test (MFF) constructed by Kagan: Reflectives are those with longer latency before making the first choice response and fewer errors in matching; impulsives are those with shorter latency and more errors. Therefore, the generality of reflection-impulsivity depends, operationally, upon what are represented by MFF latency and error measures. In other words, what is to be examined is whether the MFF latency and errors correlate with the corresponding measures derived from other " problemsolving situations with response uncertainty" (Kagan \& Messer, 1975).

Among such situations, conceptual matching tasks seem especially challenging. Recently, several investigators have found interesting differences in response patterns or "strategies" between MFF reflectives and impulsives in conceptual matching or identification tasks, such as "20-questions", "pattern matching", etc., where the number of instrumental responses is countable and has a fixed 
necessary minimum. Ault (1973) found that in the 20-questions task 1st-, 3rd- and 5 th-grade reflectives made cognitively more mature constraint-seeking questions mentioning a common feature of 2 or more alternatives more often, and questions directly naming only one of the alternatives less often, than impulsives. Denney (1973) also found that among 7 8-year-olds MFF latency correlated positively with the percentage of constraintseeking responses in the 20 -questions task and another task of identification through questioning. Furthermore, McKinney's earlier study (1973) revealed that 2ndgrade reflectives tended to use constraintseeking (his "focusing") strategy more often, and scanning and random strategies less often than impulsives, thus extracting a larger amount of information per question. His later study (McKinney, 1975) successfully replicated these findings among 7-, 9- and 11-year-olds with a pattern matching task where necessary information was obtained non-verbally as well as with various 20 -questions tasks.

Though all of these investigators used the term "strategy", their findings can be described in terms of the amount of instrumental information-seeking as well: Reflectives tend to make more instrumental responses (responses other than giving a possible answer) preceding the first-choice response (giving a possible answer). This is quite consistent with the Kagan-Kogan conceptualization of reflection-impulsivity. However, none of them has examined the generality of the dimension among kindergarten children. As Kagan and Messer (1975) suggested, this dimension may correlate with other variables differently among younger children than with those obtained with older children. Therefore, it is worth examining whether MFF measures predict response patterns in conceptual matching tasks among kindergarteners.

No studies with young children have been reported probably because both the 20-questions task and the Neimark-Lewistype pattern-matching task are too hard for kindergarteners. However, with modifications, we can apply conceptual matching tasks to young children. If we use the referential communication task with the child as receiver, where we can easily distinguish the verbal question-asking from the nonverbal choice (button-push) response, we can assess the child's tendency to seek instrumental information before making a decision. Though the Neimark-Lewis-type task is too hard, we can use Olson's task, which has been given even to 4-year-olds (Olson, 1966), and similarly assess the kindergartener's tendency to seek information (represented as the number of moves) before making a choice.

Two conceptual matching tasks, namely, RG and CPM (see below for description), were thus adopted in this study. In addition to MFF, another perceptual matching task (TVM) was used. TVM, involving tactual-visual matching, was similar to Kagan's HVM (Haptic-visual matching), which had shown a highly significant correlation with MFF among 158 1st-grade children (Kagan, Pearson, \& Welch 1966).

We predicted the following pattern of significant correlations, assuming the generality of the dimension of reflectionimpulsivity:

\begin{tabular}{c|c|c|c}
\hline & \multicolumn{3}{|c}{ Conceptual } \\
\cline { 2 - 4 } & $\begin{array}{c}\text { No. of } \\
\text { information- } \\
\text { seeking } \\
\text { responses }\end{array}$ & $\begin{array}{c}\text { No. of } \\
\text { guesses }\end{array}$ & $\begin{array}{c}\text { No. of } \\
\text { erroneous } \\
\text { choices }\end{array}$ \\
\hline \begin{tabular}{c|c} 
Lerceptual : \\
Latency
\end{tabular} & + & - & - \\
\hline
\end{tabular}

However, expected correlations do not necessarily mean that the dimension of reflection-impulsivity exists as an individual trait across developmental levels. Though the term "cognitive style" im- 
plies that it is orthogonal to ability or to the level of development, reflectives in fact tend to be cognitively more mature and to perform better than impulsives, at least up to certain age levels, because children become more and more reflective as they grow older until the 4th- or 5th-grade (Kagan, 1966). As Kagan and Messer (1975) indirectly admit, the MFF measures in those age levels represent cognitive developmental maturity as well as an individual trait of reflection-impulsivity, and the former may explain the apparent cognitive style differences in conceptual strategy found in the previous studies, where, as was aptly pointed out by Achenbach and Weitz (1975), MA was not controlled. It should be noted that cognitively mature subjects tend to adopt constraint-seeking strategy (Mosher \& Hornsby, 1966; Olson, 1966; Eimas, 1970; Neimark \& Lewis, 1967).

The generality of reflection-impulsivity as a trait should be examined with partialing out at least CA, or at most MA. Controlling CA may cause underestimation of the contribution of developmental variance, whereas partialing out MA or the intelligence test raw score may cause its overestimation, because the tendency to "reflect on" the correctness of the answer will be advantageous on some intelligence test items. Therefore, if we find significant residual correlations in the expected direction among measures of predecision information-seeking responses and of erroneous choice responses after $\mathrm{MA}$ is kept constant, we can safely conclude there exists a "general" trait of reflection-impulsivity even among young children. Thus, regarding MFF measures as primarily those of cognitive maturity, as is often suggested, is not warranted. If most significant correlations disappear when CA is partialed out, however, we must conclude that MFF essentially measures the level of cognitive development or maturity instead of cognitive "style", at least among young children.
Finally, if most of the resulting partial correlations remain significant with CA controlled but become insignificant with MA controlled, our conclusion as to the existence of the trait of reflection-impulsivity must be withheld, since developmental and trait variances cannot clearly be separated.

\section{Method}

\section{Tasks}

Four matching tasks, each of which required the child to seek some information in order to give the correct answer with confidence, were given. MFF and TVM were concerned with perceptual matching, where the probability of making correct responses tended to increase gradually with an increasing amount of information, while RC and CPM were concerned with conceptual matching, where the minimum necessary number of information-seeking responses was fixed. RC required informationseeking in a social-interpersonal situation, CPM in a physical situation. Verbal intelligence test items from a Japanese version of StanfordBinet were also given to obtain a crude estimate of MA.

$M F F$. The standard administration procedure for MFF was followed. Mean latency to first responses (in sec) and the total number of errors were calculated as usual.

Tactual-Visual Matching (TVM). This task required a child to match information from two modalities (tactual and visual). The child was asked to identify a geometric shape which was identical to the target, from four similar alternatives visually presented, by handling the target shape, fixed on a piece of cardboard and concealed in a box (see Fig. 1).

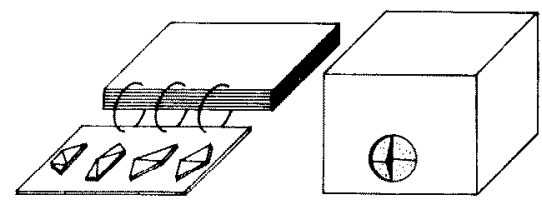

Fig. 1. A box containing the target and four alternative shapes (TVM). 
Those shapes were $10.8-24.5 \mathrm{~cm}$ in size with 3-7 straight sides, and made of $3 \mathrm{~mm}$ thick plastic. There were eight items. The four alternatives in each item were differently shaped but of nearly equal size, and so could be distinguished in terms of the number of turns, the number and configuration of acute angles, long sides, etc. If the child's response was correct, the experimenter pulled out the hidden target shape and showed it to the child, saying, "That's right". If the child was wrong, the experimenter said, "That's not the right one," and urged the child to find " the one that is just like the hidden one". After the third choice response, even when it was incorrect, the experimenter showed the target shape, and proceeded to the next item. Although this task was similar to HVM devised by Kagan, it differed in that the target and four alternative shapes were presented simultaneously, so that no memory was needed. Mean latency to first responses (in sec) and the total number of errors were adopted to represent individual performance.

Referential Communication with inadequate message $(R C)$. A simplified version of "the 20-Questions Game" was given in the standard "referential communication" setting, with the apparatus devised by Dickson (1974). A child as receiver was required to choose one picture from among four which differed in two dimensions on the basis of the verbal description given by the experimenter as sender, and to push a button beneath that picture (see Fig. 2). It was necessary for the child to ask two yes-no questions about each item in order to make the correct choice with certainty, because the experimenter always gave non-differentiat-

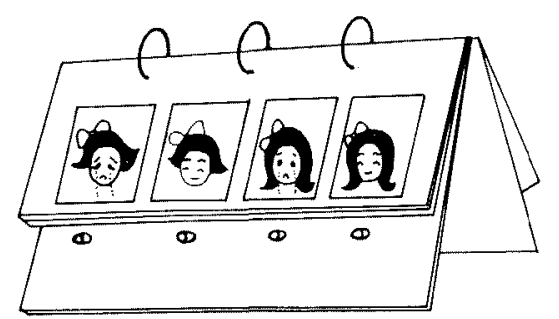

Fig. 2. Apparatus with four alternative pictures (RG). ing messages, e.g., "a girl with a ribbon" for the set in Fig. 2. The child was told to ask yes-no questions if he thought the given messages were inadequate and to push the button only after he was sure which was the right picture. Preceding the test items, three practice items were given. First, the child was asked to be a sender, i.e., to describe one of the four pictures (large or small house with a round or square window) so that the experimenter could choose it. The experimenter as receiver, irrespective of the adequacy of the child's description, asked two yes-no questions ("Is the house large [or small]?" and "Is the window round [or square]? ") before pushing the button for demonstration. This procedure was repeated with another set of pictures on the second practice item. Then, as the final practice, the child was asked to be a receiver, and the experimenter as sender gave insufficient messages. This was the only case where, if the child make a choice response without questioning, he was urged to ask questions with an intentional "That's incorrect" feedback. Then five test items were given. The child was given an answer, "Yes" or " No", by the experimenter only to his specific questions. In the case of the child's asking a nonspecific question, the experimenter repeated the initial description without adding further information. In the test items, when the child made a choice, the experimenter gave the child the feedback, "That's right" or "That's incorrect ", according to predetermined rules, irrespective of the child's question-asking. The child was allowed to make only one choice response for each item. The number of questions asked, the number of errors and the number of guesses (i.e., choice responses without sufficient logical information) were selected to indicate the child's mode of informationseeking.

Conceptual Pattern Matching (CPM). A revised Olson's Task (Olson, 1966) was used. Stimulus patterns consisted of red and black squares arranged in a $3 \times 3$ matrix on a $12.5 \times 17 \mathrm{~cm}$ card. The problem cover, made of cardboard, had 9 windows, each of which could be opened. The child was asked to find which of the two- 
TABLE 1

Correlations among MFF/TVM measures $(n=51)$

\begin{tabular}{l|c|c|c|c|c}
\hline & $\begin{array}{c}\text { MFF } \\
\text { errors }\end{array}$ & $\begin{array}{c}\text { TVM } \\
\text { latency }\end{array}$ & $\begin{array}{c}\text { TVM } \\
\text { errors }\end{array}$ & CA & $\begin{array}{c}\text { verbal } \\
\text { ability }\end{array}$ \\
\hline MFF latency $(m=11.3 ; S D=9.3)$ & $-.52^{* *}$ & $.40^{* *}$ & $-.28^{*}$ & .08 & $.30^{*}$ \\
MFF errors $(m=17.0 ; S D=7.8)$ & & $-.28^{*}$ & $.34^{* *}$ & -.07 & $-.49^{* *}$ \\
TVM latency $(m=12.4 ; S D=5.3)$ & & & $-.35^{* *}$ & .08 & .01 \\
TVM errors $(m=4.2 ; S D=2.6)$ & & & & .07 & $-.46^{* *}$ \\
* significant at $5 \%$ level (one-tailed test) & & & & \\
** significant at $1 \%$ level (,$\%(2)$
\end{tabular}

or the four-alternative patterns presented was hidden under the problem cover, by opening windows (see Fig. 3). The child was allowed to seek as much information as he liked by opening windows, but was told to make a choice as soon as he found the solution. (This statement was repeated from time to time.) After two practice items, where, if the child opened only one window, he was again told that he was allowed to open more until he was sure which pattern was hidden, four test items, one with two alternatives and the others with four, were given. The child was allowed to make only one choice response, after which the experimenter removed the problem cover to show the hidden pattern to the child, and also gave verbal feedback. The number of "moves" (window openings), errors, and guesses were taken as indices.

Verbal ability items. Four verbal items from the Tanaka-Binet intelligence test (a Japanese version of Stanford-Binet), all for the age range 6-8 years, were selected in order to give a rough estimate. They were 'opposite analogies II \& III', ' picture absurdities I', and 'memory for sentences'. They were administered and scored as described in the manual (so individual scores varied from $0-4$, with
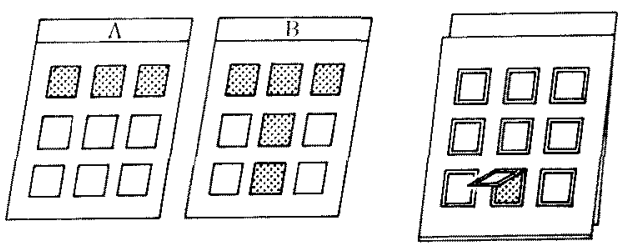

Frg. 3. Problem cover with windows (right) and two alternative patterns (CPM). mean of 2.7 and standard deviation of 1.0 ).

\section{Data Collection Procedure}

Data were gathered as a part of a larger study on information-seeking in young children, where subjects were given 7 individual test sessions over a period of 8 months. The data from 51 kindergarten children (25 boys and 26 girls) who received all the tasks described above will be analyzed here. They were the relatively older pupils of 5-6-year-olds' classes, and ranged in age from 67 to 73 months (mean= $70.3, S D=1.9$ ) when they were given the first test session.

MFF was given in the first session, $\mathrm{RC}$ in the second and CPM in the third (both 4 months later), TVM in the fourth (another month later) and verbal ability items in the fifth (further 2 months later).

Two female graduate students served as experimenters, and each administered some tasks to all the subjects.

\section{Results}

First, consistencies between two perceptual matching tasks were examined. Correlations among latency and error measures of MFF and TVM were all significant in the expected direction (by one-tailed test, since we had had clear expectations about the sign of each correlation): Within the latency/error measures the correlations were positive, and between them the correlations were negative (see Table 1). This was consistent with the findings by Kagan, et al. (1966) 
TABLE 2

Gorrelations between MFF/TVM and RC/CPM measures $(n=51)$

\begin{tabular}{|c|c|c|c|c|c|c|}
\hline \multirow{2}{*}{1} & \multicolumn{3}{|c|}{$\mathrm{RC}$} & \multicolumn{3}{|c|}{ CPM } \\
\hline & $\begin{array}{l}\text { Questions } \\
(m=5.6 \\
S D=4.0)\end{array}$ & $\begin{array}{c}\text { Guesses } \\
(m=3.0 \\
S D=2.0)\end{array}$ & $\begin{array}{c}\text { Errors } \\
(m=2.0 \\
S D=1.6)\end{array}$ & $\begin{array}{c}\text { Moves } \\
(m=28.6 \\
S D=15.2)\end{array}$ & $\begin{array}{c}\text { Guesses } \\
(m=1.0 \\
S D=1.3)\end{array}$ & $\begin{array}{c}\text { Errors } \\
(m=0.8, \\
S D=0.9)\end{array}$ \\
\hline MFF latency & -.05 & -.02 & .15 & .06 & -.18 & $-.29 *$ \\
\hline MFF errors & $-.34 * *$ & $.40^{* *}$ & $.29^{*}$ & .03 & .16 & .18 \\
\hline TVM latency & .22 & -.18 & -.14 & $.27^{*}$ & -.20 & -.19 \\
\hline TVM errors & $-.38 * *$ & $.37^{* *}$ & .19 & $-.34 * *$ & $.38 * *$ & .19 \\
\hline $\mathrm{CA}$ & -.07 & .10 & .07 & -.13 & .17 & -.06 \\
\hline Verbal ability & $.30^{*}$ & $-.34^{*}$ & -.21 & .08 & -.25 & -.10 \\
\hline
\end{tabular}

that MFF and HVM correlated highly in the expected direction. These measures correlated only negligibly with CA, probably because of the very limited range of CA. Partialing out the effect of CA did not cause any remarkable changes in the correlation patterns. However, partialing out verbal ability left only 4 out of the 6 correlations still significant, eliminating TVM errors $\times$ MFF latency and TVM errors $\times$ MFF errors (using one-tailed $t$-test). These consistencies suggest that these two time-measuring, perceptual matching tasks can be regarded as equally revealing of children's reflection-impulsivity, though their error measures may represent verbal ability or cognitive maturity in general as well.

Therefore, the above four measures were correlated with the measures obtained in the conceptual matching tasks (RC and CPM). These are shown in Table 2. Of the 24 correlations, 21 had the expected signs $(+,-)$ and 9 were significant in the expected direction (also by one-tailed test). In other words, reflectives, who were revealed by longer latency and fewer errors on MFF and TVM, tended to make more informationseeking responses and fewer erroneous choice responses on $\mathrm{RC}$ and CPM than impulsives. Moreover, against the standard of minimum necessary information, reflectives tended to make fewer guessing responses (i.e., choices with insufficient information).

Though latency measures were generally poorer predictors of $\mathrm{RG}$ and $\mathrm{CPM}$ measures than the error measures, probably because none of the "conceptual" measures were time measures whereas some were error measures, their correlation patterns also tended to be in the expected direction.

Partialing out CA did not cause any significant changes in correlation patterns. Partialing out verbal ability did not change the pattern of correlations either: 7 out of the 9 (excluding MFF errors $\times$ $\mathrm{RC}$ questions and MFF errors $\times \mathrm{RC}$ errors) remained significant (also by onetailed $t$-test).

\section{Discussion}

The above findings suggest that longer latency and fewer errors on MFF and TVM tended to be associated with tendency to seek more information before decision-making in conceptual matching tasks, though this association was far from perfect and could be explained in part by shared covariance with MA or verbal ability. Considering that (1) these tasks were given in several different test sessions over 5 months, and 2) the number of items 
in $\mathrm{RC}$ and CPM were very small, thus making the measures from them less reliable, it can be said that the 5-6-year-old children had some "general" trait to seek much information before making a decision. In other words, the present findings lend support to the KaganKogan conceptualization of reflectionimpulsivity: The tendency to reflect on the validity of a solution hypothesis in varied situations having response uncertainty. This dimension does not primarily represent cognitive maturity.

This was also confirmed by analysis of the same data in another way (Inagaki \& Hatano, 1975). Eighteen reflectives classified by the traditional double median method using MFF latency and errors, differed in the expected direction from 18 impulsives on all of the 6 measures of RC and CPM, i.e., the reflectives tended to make more instrumental informationseeking responses, fewer guesses, and fewer errors. Out of the 6 comparisons, 3 (RC questions and guesses, and CPM errors) were significant at a $5 \%$ level by onetailed $t$-test.

Why, then, were 3 correlations (MFF latency by $\mathrm{RG}$ questions, MFF latency by RG errors, and MFF errors by CPM moves) out of the 24 in Table 2 in the unexpected direction? This was probably due not only to the limited generality of the dimension measured by MFF, but also to the additional influence of factors other than reflectivity on the amount of information-seeking in the conceptual matching tasks. Shyness may have influenced the number of questions asked in RC. One extremely reflective girl, who spent more than $70 \mathrm{sec}$ on the average for each matching in MFF with only 4 errors, did not ask any questions in RC and made no correct responses. Since she showed exceptionally long latency before making a choice on RC (64 sec on the average), we think she did know that she needed more information, but hesitated to ask questions probably because of her shyness.
Excluding her data, correlation of MFF latency with $\mathrm{RC}$ questions was .15 , and that with RC errors, -.09 , both in the expected direction. On the other hand, the large number of moves in CPM may have been due to pleasure in the act of opening, rather than to the desire to be accurate. Some children, who were impulsive on MFF and TVM, seemed to be interested more in opening windows than in identification of the hidden alternative in CPM. After the exclusion of two children of this type, the correlation between MFF error and CPM moves became -.08 , again in the expected direction.

Cognitive style differences in the efficiency of information processing were also examined. For each subject, "the amount of information obtained divided by the number of moves' (in CPM) and 'the amount of information obtained divided by the number of questions' (in $\mathrm{RC}$ ) were calculated. Of the 8 correlations between these two efficiency measures on one hand, and the latency and error measures of MFF and TVM on the other, 5 were in the direction opposite from the expectation that reflectives would tend to process information more efficient$l y$, and none of the remaining 3 in the expected direction were significant. Two possible explanations of these findings can be suggested: (1) Since probably only very few of our 5-6-year-olds used any systematic strategy, impulsive decisionmaking after a few information-seeking responses may have resulted in higher efficiency; (2) Being excessively reflective may not have been efficient because Japanese children are known to be much more reflective than their US counterparts (Hatano, 1974). Anyway, the superiority of reflectives over impulsives in efficiency of information-processing, as found in McKinney (1973, 1975), is limited to specific age levels and/or cultures, while reflectives' making more instrumental information-seeking responses than impul- 
sives, which is implied by the conception of reflection-impulsivity, is more general in nature.

\section{REFERENGES}

Achenbach, T. M., \& Weisz, J. R. 1975 Impulsivity-reflectivity and cognitive development in preschoolers: A longitudinal analysis of developmental and trait variance. Extended report of article that appeared in Developmental Psychology, 11, 413-414.

Ault, R. L. 1973 Problem-solving strategies of reflective, impulsive, fast-accurate, and slowinaccurate children. Child Development, 44, 259-266.

Denney, D. R. 1973 Reflection and impulsivity as determinants of conceptual strategy. Child Development, 44, 614-623.

Dickson, W. P. 1974 An instructional device for teaching verbal skills through structured interaction between children in a communication game: Final report (National Institute of Education Project No. 1-I-101). Stanford, Ca.: Stanford University, School of Education.

Eimas, P. D. 1970 Information processing in problem solving as a function of developmental level and stimulus saliency. Developmental Psychology, 2, 224-229.

Hatano, G. 1974 The development of reflectivity. Development of children and education. (Report No. 2) Tokyo: National Institute for Educational Research. (in Japanese)

INAGAKI, K., \& HATANo, G. 1975 Information-seeking behavior in young children. Paper presented at the 17 th Annual Convention of the Japanese
Association of Educational Psychology, Sendai. (in Japanese)

Kagan, J. 1966 Developmental studies in reflection and analysis. In A. H. Kidd \& J. L. Rivoire (Eds.), Perceptual development in children. New York: International University Press.

KAgAN, J., \& KogAN, N. 1970 Individual variation in cognitive processes. In P. Mussen (Ed.), Carmichael's mannual of child psychology. New York: Wiley.

KagAN, J., \& Messer, S. B. 1975 A reply to "Some misgivings about the Matching Familiar Figures Test as a measure of reflection-impulsivity". Developmental Psychology, 11, 244248.

Kagan,J., Pearson, L., \& Welch, L. 1966 Conceptual impulsivity and inductive reasoning. Child Development, 37, 583-594.

McKinney, J. D. 1973 Problem-solving strategies in impulsive and reflective second graders. Developmental Psychology, 8, 145.

McKinney, J.D. 1975 Problem-solving strategies in reflective and impulsive children. Journal of Educational Psychology, 67, 807-820.

Mosher, F. A., \& Hornsby, J. R. 1966 On asking questions. In J.S. Bruner, R. R. Olver, \& P. M. Greenfield (Eds.), Studies in cognitive growth. New York: Wiley.

NeImArk, E. D., \& Lewts, N. 1967 The development of logical problem-solving strategies. Child Development, 38, 107-117.

OLson, D. R. 1966 On conceptual strategies. In J. S. Bruner, R. R. Olver, \& P. M. Greenfield (Eds.), Studies in cognitive growth. New York: Wiley.

(Received Nov. 29, 1976) 